\title{
Metabolic consequences of second-generation antipsychotics in youth: appropriate monitoring and clinical management
}

This article was published in the following Dove Press journal:

Adolescent Health, Medicine and Therapeutics

26 September 2014

Number of times this article has been viewed

\section{Rebecca A Krill}

Sanjiv Kumra

Department of Psychiatry, University of Minnesota, Minneapolis, MN, USA

Correspondence: Sanjiv Kumra Department of Psychiatry, University of Minnesota, F-282/2A West, 2450 Riverside Avenue, Minneapolis, MN 55454, USA

Email kumra002@umn.edu
Objective: To review the metabolic consequences of second-generation antipsychotics in youth and current monitoring and intervention guidelines for optimal treatment.

Background: Second-generation antipsychotics have largely replaced the use of first-generation antipsychotics in treating psychotic disorders in youth. In addition, there has been a dramatic increase in using these medications to treat a variety of nonpsychotic disorders. These medications have significant metabolic side effects, including weight gain. This raises concern, given the problem of pediatric obesity.

Materials and methods: A review of current literature looking at prescribing practices and possible reasons for the increased use of second-generation antipsychotics in children and adolescents was conducted. Review of the mechanisms for why youth may be particularly vulnerable to the metabolic consequences (particularly weight gain) was similarly completed. In addition, data supporting the efficacy, rationale, and unique side-effect profile of each individual second-generation drug were evaluated to help inform providers on when and what to prescribe, along with current monitoring practices. The current evidence base for possible interventions regarding the management of antipsychotic-induced weight gain was also evaluated.

Results and conclusion: On the basis of the literature review, there are several speculated reasons for the increase in prescriptions of second-generation antipsychotics. The choice of antipsychotic for youth should be based upon the disorder being treated along with the unique side-effect profile for the most commonly used second-generation antipsychotics. Monitoring strategies are also individualized to each antipsychotic. The current interventions recommended for antipsychoticinduced weight gain include lifestyle management, switching medication to a drug with a lower propensity for weight gain, and pharmacologic (particularly metformin) treatment.

Keywords: antipsychotics, children, youth, metabolic, weight gain

\section{Introduction}

There has been a dramatic increase in prescription of second-generation antipsychotics (SGAs) for children and adolescents. ${ }^{1,2}$ The clinical utility of these medications has been demonstrated for a number of psychiatric disorders affecting youth, including autism, schizophrenia, bipolar disorder, and Tourette's syndrome, and in clinical practice these medications are routinely prescribed off-label for the management of aggression in children with externalizing behaviors. ${ }^{1-3}$ Unfortunately, these medications are also associated with significant side effects, including risk for prolactin elevation, extrapyramidal movements, tardive dyskinesia, effects on cardiological function, weight gain, and metabolic disorders. ${ }^{4}$ Of these side effects, weight gain and metabolic disorders are highly prevalent, difficult to manage, and could have potential long-term consequences. ${ }^{5}$ Although some guidelines have been developed for the monitoring 
of these adverse events, adherence to these guidelines by practitioners has been suboptimal. ${ }^{6}$ In addition, there have been few evidence-based approaches developed to manage these side effects. ${ }^{7}$ However, in response to the alarming increase in antipsychotic prescriptions for youth in Canada, a panel entitled Canadian Alliance for Monitoring Effectiveness and Safety of Antipsychotics in Children (CAMESA) was formed to review systematically the side-effect burden of the most common SGAs. ${ }^{8}$ The expert panel made their findings and monitoring/management recommendations available to the public. In efforts to summarize concisely and build upon the current knowledge base, the purpose of this article is threefold: 1) review available data suggesting that youth may be particularly susceptible to development of metabolic side effects, 2) review the efficacy and safety profile of the most commonly prescribed SGAs to guide the initial choice in prescribing, and 3) encourage utilization of current monitoring and management guidelines suggested by CAMESA and discuss potential interventions to combat metabolic side effects (specifically weight gain).

\section{Clinical scenario}

Jordan is a 16-year-old boy admitted to the inpatient child and adolescent psychiatric unit after his family notices a decline in his functioning over the past several months. He has become increasingly withdrawn, with poor hygiene, and he is afraid that his food is being poisoned. Upon admission, he is aggressive with providers, and reports somatic complaints and delusions that he may in fact already be dead. His admission weight and body mass index (BMI) are $145 \mathrm{lb}(65.77 \mathrm{~kg})$ and 22.7, respectively. He is started on an SGA, and over the course of several weeks he is less paranoid, his somatic complaints have diminished, and he is discharged to a lower level of care to help him transition back to school. Parents continue reporting sustained improvement to the outpatient psychiatrist. Ten months after his initial hospitalization, Jordan has gained 20 pounds, with a corresponding increase in BMI, and his lipid panel reveals diminished high-density lipoprotein. He reports feeling a constant insatiable hunger. His parents report, "We finally feel like we have our son back, but what should we do about his weight gain?"

\section{Trends in antipsychotic use for youth with severe emotional disturbances}

The US Food and Drug Administration (FDA) has approved antipsychotics for use in several disorders in children and adolescents that include schizophrenia, bipolar disorder,
Tourette's syndrome, and associated aggression/irritability in autism-spectrum disorder. SGAs largely replaced firstgeneration antipsychotics (FGAs) in the past two decades, due to their perceived lower risk for acute and chronic extrapyramidal side effects (EPSEs), for which youth are thought to be more susceptible than adults. ${ }^{9}$ EPSEs include akathisia, parkinsonism, acute and tardive dystonia, and tardive dyskinesia. The introduction of SGAs did not fully ameliorate these side effects, but diminished them greatly, due to their lower $\mathrm{D}_{2}$-receptor affinity and faster dissociation from the $\mathrm{D}_{2}$ receptor. ${ }^{10}$

Today, antipsychotic prescriptions are among the most commonly prescribed medications. ${ }^{1,2}$ For every 100 officebased visits in 2005-2009, an antipsychotic was prescribed at a rate of 1.83 for a child and 3.76 for an adolescent. ${ }^{1}$ There are several probable reasons for this increase, which are outlined in a recent paper by Correll et al. ${ }^{11}$ There is a greater recognition that severe mental disorders frequently begin in childhood and adolescence, are chronic in nature, and require long-term medication use. As such, there has been a subsequent increase in the evidence base and indications for antipsychotic use in this population. Another possible contributor has been the overreliance on pharmacologic management to achieve symptom reduction in the current environment, which focuses on expedience and cost, particularly for inpatient settings. This is coupled with a regional shortage of services and trained professionals in psychosocial interventions, especially for behaviorally challenged children. The substantial proportion of antipsychotic use in youth with attention deficit/hyperactivity disorder (ADHD) and other disruptive behavioral disorders could be a consequence of this.

Specific trends for antipsychotic use in youth reflect these challenges. A recent study by Birnbaum et al found that from the early 1990 s through the late 2000 s, $30.5 \%( \pm 18.5 \%)$ of antipsychotic treated youth had a diagnosis of ADHD. ${ }^{2}$ This was secondary only to those with disruptive behavioral disorders $(31.3 \% \pm 22.6 \%)$, followed by mood disorders $(24.0 \% \pm 27.3 \%)$ and psychotic disorders $(8.8 \% \pm 5.9 \%) .{ }^{1}$ For adolescents, the corresponding rates were highest for bipolar disorder not otherwise specified (14.9\%), anxiety disorder not otherwise specified (12.6\%), and ADHD (11.4\%). ${ }^{1}$ Overall, there was also a marked increase in antipsychotic visits in youth with a diagnosis of mood disorder $(31.3 \%$ in 2005-2009 versus 6.6\% in 1993-1998). ${ }^{1}$ A similar trend was found in youth carrying an anxiety disorder (16.3\% versus $5.3 \%) .{ }^{1}$ The most common SGAs prescribed for children from 2005 to 2009 were risperidone $(42.1 \%)$, aripiprazole $(28.0 \%)$, 
quetiapine (19.2\%), and olanzapine (4.4\%). For adolescents, aripiprazole was the most commonly prescribed (29.0\%), followed by quetiapine (26.8\%), risperidone $(23.0 \%)$, and olanzapine $(9.3 \%){ }^{1}$

\section{Susceptibility for youth to develop antipsychotic-induced weight gain}

In spite of SGAs' efficacy, it has only been in the past decade that their metabolic side-effect profile has been carefully studied in adults, and much less so in youth. ${ }^{12}$ Extension of adult findings toward guiding clinical practice in children and adolescents may not be appropriate. Compared with adults, children have rapid tissue growth, higher ratio of liver organ to tissue mass, greater extracellular tissue water and glomerular filtration rates, lower protein binding, reduced fat-tissue mass, and more active hormone release in adolescence. ${ }^{13}$ Thus, children may require different dosing strategies and experience more frequent and unique side effects than adults.

In a large naturalistic cohort study conducted from 2001 to 2007 entitled Second-Generation Antipsychotic Treatment Indications, Effectiveness and Tolerability in Youth (SATIETY), data collected from antipsychotic-naïve youth demonstrated weight gain over the first 3 months of treatment with all SGAs in the following order from most weight gain to least: olanzapine $(8.5 \mathrm{~kg})>$ quetiapine $(6.1 \mathrm{~kg})>$ risperidone $(5.4 \mathrm{~kg})>$ aripiprazole $(4.4 \mathrm{~kg}){ }^{12}$ This is in contrast to psychiatrically ill youth who refused or stopped SGA treatment within 4 weeks and gained only $0.2 \mathrm{~kg}$. De Hert et al completed a systematic review of placebo-controlled studies of SGAs in youth regardless of diagnosis and abstracted data from over 3,000 patients. Based on the results, the weight-gain potential appeared similar to the SATIETY results: olanzapine $\geq$ clozapine $>$ risperidone $\geq$ quetiapine $>$ aripiprazole $=$ ziprasidone. $^{14}$

In the SATIETY study, SGA-treated youth gained $\geq 7 \%$ of their baseline weight within the first few months, with continued weight gain indefinitely. This finding is even more alarming against the backdrop of the current obesity epidemic in the US among children. According to the latest statistics from the US Centers for Disease Control and Prevention, approximately $17 \%$ (or 12.5 million) of children and adolescents aged $2-19$ years are obese. ${ }^{15}$

\section{Mechanisms underlying weight gain}

While the exact mechanism of weight gain and dyslipidemia from SGAs in either children or adults is poorly understood and complex, several theories have been studied and proposed. There are increasing data that dyslipidemias and glucose intolerance can occur independently of weight gain. ${ }^{12}$ In a comprehensive review of possible explanations, Maayan and Correll $1{ }^{16}$ cite research supporting the view that antipsychotics may decrease physical activity and interfere with the body's utilization of energy stores and energy expenditures. In addition, there is significant evidence that points to increased caloric intake as the major cause of weight gain. Mechanisms for the increased appetite and decreased satiety may involve complex interactions with serotonergic, dopaminergic, and histaminergic receptors and hypothalamic peptides and hormones involved in energy homeostasis that are perturbed by antipsychotic medications. This may explain in part why medications with strong hypothalamic $\mathrm{H}_{1}$-receptor affinity are correlated with a higher propensity for weight gain.

There is concern that children and adolescents may be at greater risk for unhealthy weight gain with SGAs than adults. ${ }^{17}$ Weight susceptibility in youth may result from differences in drug metabolism and immaturity of the prefrontal cortex compared to adults, resulting in decreased top-down regulation of the hypothalamus. This could lead to increased appetite and caloric intake. ${ }^{16}$ It also may be the result of an artifact of prior drug treatment. Youth enrolled in clinical trials often have less prior exposure to antipsychotic medications than adults with chronic illness, who likely have longer durations of antipsychotic exposure, leading to an attenuated signal in registered trials. ${ }^{16}$ This hypothesis is supported by similar findings found in first-episode adults with psychosis, who were observed to have a more significant propensity to gain weight compared to adults with chronic mental illness. ${ }^{12}$

\section{Initial selection of antipsychotic medication}

One of the most important decisions providers make is selecting which antipsychotic to initiate. Most of the evidence base for SGAs in youth comes from research in psychotic disorders, bipolar disorder, and disruptive behavior disorders (including autism-associated irritability and conduct disorder), with the majority of studies looking at risperidone in the latter category. SGAs have also been examined in the treatment of Tourette's syndrome. These studies were conducted in populations with varying levels of illness severity (inpatient, outpatient, or combination). Also, while the rating scales used to measure symptomatic improvement proved to be statistically significant in these studies, one should keep in mind how this translates clinically. 
With the exception of clozapine, systematic analysis trends toward superiority of SGAs over FGAs in terms of symptomatic improvement, ${ }^{18}$ but as mentioned earlier, FGAs have largely fallen out of favor due to EPSEs. There are a few comparative studies of nonclozapine SGAs in treating psychotic disorders in youth, and they all appear to perform similarly. ${ }^{3,19}$

For bipolar disorder, all SGAs likewise appear the same in terms of efficacy when compared with placebo. ${ }^{3,19,20}$ There are few head-to-head studies looking at conventional mood stabilizers (anticonvulsants/lithium) versus SGAs for bipolar disorder. Limited evidence thus far however points to SGAs as superior for pediatric mania, based on meta-analysis when compared to placebo. ${ }^{21}$ The authors of the study cautioned, however, that SGAs have a greater side-effect burden than mood stabilizers. The use of SGAs for bipolar depression is unclear, as some studies have demonstrated no difference in depressive symptoms when comparing SGAs to placebo. ${ }^{3}$

Unfortunately, there is a dearth of comparative studies investigating pharmacologic versus nonpharmacologic treatments alone and in combination for behaviorally disruptive children. Likewise, there are no studies in youth directly looking at SGA use for anxiety, nor the commonly diagnosed mood disorder not otherwise specified. Clinicians are often left to rely heavily on anecdotal experience and/or evidence from adult studies to make treatment decisions. In addition, there are few studies looking at SGAs for Tourette's syndrome. Meta-analysis supported the use of risperidone and ziprasidone, with moderate strength of evidence for both. ${ }^{3}$

Clinicians ought to base their choice on efficacy (balancing both the evidence base with clinical experience), a well-established safety profile, and data supporting the long-term use of a medication to guide informed consent. In the following sections, we review data supporting the effectiveness of the most commonly used SGAs in youth.

\section{Risperidone}

Risperidone is approved by the FDA for treating schizophrenia in adolescents aged 13-17 years, bipolar disorder in those 10-17 years, and autism-related aggression/irritability in children 5-17 years of age. In Europe, it is approved for children with aggression in conduct disorder. It was the most often-prescribed SGA in the longitudinal Child and Adolescent First Episode Psychosis Study (CAFEPS), followed by olanzapine and quetiapine in children 9-17 years of age. ${ }^{22}$

Approval to treat adolescent schizophrenia came from two short-term, double-blind controlled trials ( 6 and 8 weeks, respectively) of patients (combination of inpatients and outpatients) experiencing acute onset of symptoms consistent with schizophrenia. ${ }^{23,24}$ Both studies demonstrated superiority compared to placebo $(n=36)$ and low-dose risperidone $(0.15-0.6 \mathrm{mg}, \mathrm{n}=82)$. There was no difference in outcomes in those patients taking $1-3 \mathrm{mg} /$ day $(\mathrm{n}=45)$ versus $4-6 \mathrm{mg} /$ day $(\mathrm{n}=44)$ in the first study. The dose range in the second study was $1.5-6.0 \mathrm{mg} /$ day $(\mathrm{n}=90)$. Those in the higher-dose group from the first study experienced more EPSEs, dizziness, and hypotonia. A US National Institute of Mental Health-funded comparison of risperidone with olanzapine and molindone did not find it superior to either medication in treating psychosis. ${ }^{17}$

Approval for treatment of bipolar disorder came from efficacy demonstrated in a 3-week, randomized, doubleblind, placebo-controlled, multicenter trial of patients ranging in ages from 10 to 17 years $(n=169) .{ }^{25}$ The efficacy on the primary parameter in those taking 3-6 $\mathrm{mg}$ /day $(\mathrm{n}=61)$ was comparable to those taking $0.5-2.5 \mathrm{mg} /$ day $(\mathrm{n}=50)$. When studied head to head in outpatient youth with bipolar disorder against valproic acid ( $\mathrm{n}=66,33$ in each arm), it was found to be superior to the mood stabilizer. ${ }^{26}$

Approval for the treatment of irritability associated with autism came from efficacy established in two 8-week, placebo-controlled trials in children and adolescents (aged $5-16$ years, combined study total $n=134,67$ in each arm). ${ }^{27,28}$ The primary outcome measure in both trials was the change from baseline to end point in the irritability subscale of the Aberrant Behavior Checklist (ABC)-I. The ABC-I subscale measures the emotional and behavioral symptoms of irritability in autistic disorder, including aggression toward others, deliberate self-injuriousness, temper tantrums, and quickly changing moods. In these studies, both demonstrated superiority when compared to placebo. However, there was no effect on core symptoms of autism.

In a meta-analysis of efficacy in autism-spectrum disorders (one of the FDA-cited studies above was not included ${ }^{28}$ ), risperidone repeatedly demonstrated improvements in the ABC-I, Autism Rating Scale, and obsessivecompulsive symptoms compared to placebo. ${ }^{3}$ When compared head to head with haloperidol, there was no significant difference on the ABC-I. In placebo-controlled relapse-prevention studies (up to 1 year), risperidone was superior to placebo in maintaining efficacy in the ABC-I subscore. In spite of the proposed benefit, the authors of the analysis concluded that overall the strength of evidence in these studies was low. ${ }^{3}$ Most recently, when studied head to head against aripiprazole ( $\mathrm{n}=59$ total), neither outperformed the other. ${ }^{29}$ 
In a meta-analysis looking at effectiveness of SGAs for aggressive behaviors associated with oppositional defiant disorder and conduct disorder, all but one study included risperidone against placebo. Moderate strength of evidence was found for risperidone, based upon greater improvement on various measures of behavior symptoms and the Clinical Global Impression scale compared with placebo, but it did not differ significantly for aggression. ${ }^{3}$

In a meta-analysis, risperidone had a significantly higher rate of EPSEs, with a corresponding odds ratio of 3.55 and corresponding higher rates of treatment with anticholinergic medications. ${ }^{8}$ In regard to metabolic consequences, a metaanalysis of randomized controlled trials (RCTs) with risperidone lasting less than 12 weeks demonstrated a $1.72 \mathrm{~kg}$ weight increase compared to placebo, with continued weight gain in RCTs lasting 6 months $(2.09 \mathrm{~kg})$ and up to 2 years. ${ }^{8}$ There was a mean increase in waist circumference of $5.1 \mathrm{~cm}$ and an increase in triglyceride levels after 10.8 weeks of therapy. ${ }^{8}$ There are case reports of risperidone associated with diabetes and hyperglycemia in children. ${ }^{8}$ Risperidone also demonstrated a significant increase in prolactin levels that appeared to then decrease over time. ${ }^{8}$ Adverse events related to hyperprolactinemia are much more common in girls than boys. ${ }^{20}$ In a more recent study (2013) by Margari et al, risperidone was studied in an open-label observational study lasting 6 months to verify tolerability in antipsychoticnaïve children ( $\mathrm{n}=22$ ) ages $6-17$ years. Results demonstrated a significant increase in weight, BMI, BMI percentile, and normal weight distribution ( $z$ score), with a trend demonstrating increases in prolactin, glucose, cholesterol, and liver-enzyme levels. ${ }^{30}$

\section{Aripiprazole}

Aripiprazole is a partial $\mathrm{D}_{2} / \mathrm{D}_{3}$ and serotonin 5- $\mathrm{HT}_{1 \mathrm{~A}}$ agonist approved for pediatric schizophrenia (13-17 years, FDA), bipolar disorder (10-17 years, FDA; $>13$ years in Europe), and irritability associated with autism-spectrum disorders (6-17 years, FDA). It is sometimes referred to as a "thirdgeneration antipsychotic", due to its differing mechanism of action than other SGAs. ${ }^{20}$

The approval for use in pediatric schizophrenia came from one 6-week, placebo-controlled trial of outpatients ( $\mathrm{n}=302,13-17$ years) comparing two fixed doses of $10 \mathrm{mg}$ and $30 \mathrm{mg} .{ }^{31}$ Though no differences were found between these two dosages, both were superior to placebo.

Approval for the treatment of bipolar I disorder in pediatric patients (10-17 years of age) was evaluated in one 4-week, placebo-controlled trial $(n=296)$ of outpatients, using two fixed doses of $10 \mathrm{mg} /$ day or $30 \mathrm{mg} /$ day. ${ }^{32}$ Again, both doses were superior to placebo, with no difference in efficacy between the two. Thus far, there do not appear to be any comparative studies of aripiprazole with other SGAs for bipolar disorder.

The efficacy of aripiprazole in the treatment of irritability associated with autistic disorder was established in two 8-week, placebo-controlled trials in pediatric patients $\left(n=316\right.$ combined, $6-17$ years of age).$^{33,34}$ Both trials demonstrated significant change from baseline to end point in the Irritability subscale of the ABC. The average end-point dose in one trial was $8.6 \mathrm{mg}$. There was significant change found in all three fixed doses of 5,10, and $15 \mathrm{mg}$ in the second trial. As with risperidone, in a meta-analysis, there was overall benefit with aripiprazole, but strength of evidence was low in supporting its use. ${ }^{3}$ A very recent study of autistic patients taking aripiprazole investigated time to relapse in those randomized to either continuous use of aripiprazole or placebo $(\mathrm{n}=85)$. The authors found no statistically significant difference in time to relapse (16 weeks) during maintenance therapy. ${ }^{35}$

Compared to other SGAs (with the exception of ziprasidone), aripiprazole has the least weight gain. ${ }^{8,20}$ When compared with placebo, however, it demonstrated higher mean weight gain and increases in BMI and waist circumference $(0.85 \mathrm{~kg}$, $0.27 \mathrm{k} / \mathrm{m}^{2}$, and $5.4 \mathrm{~cm}$, respectively). ${ }^{8}$ However, increased body weight with aripiprazole may reach a plateau within 3-6 months..$^{20}$ Aripiprazole also has been found to significantly decrease prolactin levels, and like risperidone has higher odds of EPSEs when compared to placebo (odds ratio 3.70). ${ }^{8,20}$

\section{Quetiapine}

Quetiapine is approved for schizophrenia (13-17 years, FDA) and bipolar disorder (10-17 years, FDA). Efficacy for pediatric schizophrenia was established in a 6-week, double-blind, placebo-controlled, randomized, multicenter parallel-group trial of two target doses of quetiapine in pediatric patients: $400 \mathrm{mg} /$ day $(\mathrm{n}=73)$ and $800 \mathrm{mg} /$ day $(\mathrm{n}=74) .{ }^{36}$ It demonstrated superiority over placebo $(n=75)$. Head to head in a sample of 32 first-episode psychosis (FEP) patients ( $\mathrm{n}=16$ in each arm), it did not yield any superiority over olanzapine in an openlabel 6-month period, ${ }^{37}$ nor did it outperform olanzapine or risperidone in a separate comparative pilot study in children with schizophrenia ( $\mathrm{n}=21$ of 30 completed). ${ }^{38}$

Efficacy for approval in the treatment of bipolar disorder was established in a 3-week, double-blind, placebocontrolled, randomized, multicenter, parallel-group trial of two target doses in pediatric patients (10-17 years) of 400 
$\mathrm{mg} /$ day $(\mathrm{n}=95)$ and $600 \mathrm{mg} /$ day $(\mathrm{n}=98) .{ }^{39}$ It demonstrated superiority over placebo $(n=91)$. It has been studied in adolescents as an adjunct to valproic acid versus valproic acid monotherapy, and led to quicker reduction in manic symptoms at $450 \mathrm{mg} /$ day. ${ }^{40}$

A randomized controlled pilot study looked at its use in conduct disorder (dose range 200-600 mg/day). Results found improvement in clinician-assessed measures and on the parent-assessed quality-of-life rating scale, with no differences in other parent-rating or aggression scales. ${ }^{41}$

Quetiapine is known to carry more sedation and hypotension than its counterparts, presumably secondary to its affinity for histamine and $\alpha$-adrenergic receptors, respectively. ${ }^{20}$ In a meta-analysis, quetiapine was not found to carry significant risk for developing EPSEs when compared to placebo. ${ }^{8}$ Weight gain was found to increase by an average of $1.41 \mathrm{~kg}$ in RCTs lasting less than 8 weeks compared to placebo and was not dose-dependent. Evidence also points to significant changes in triglyceride levels compared to placebo. The same meta-analysis ${ }^{8}$ points to significant changes in triglyceride levels compared to placebo. In addition, trials lasting longer than 3 months report continued weight gain and increase in BMI, as well as increases in thyroid-stimulating hormone and decreases in free thyroxine. ${ }^{8}$ There are case reports of quetiapine-associated hyperglycemia and diabetes. ${ }^{8}$

\section{Olanzapine}

Olanzapine is approved for the treatment of schizophrenia and bipolar disorder in adolescents (13-17 years, FDA). Establishment of efficacy for acute treatment of schizophrenia in adolescents came from a 6-week, doubleblind, placebo-controlled, randomized trial of inpatients and outpatients with schizophrenia. ${ }^{42}$ An average dose of $11.1 \mathrm{mg} /$ day was significantly more effective than placebo. More olanzapine-treated youth $(n=71)$ completed the trial than placebo $(n=35)$.

Establishment for approval of treatment of bipolar disorder came from a 3-week, double-blind, placebo-controlled, randomized trial of adolescent inpatients and outpatients $(\mathrm{n}=107) .{ }^{43}$ As a flexible-dose trial, an average of $8.9 \mathrm{mg} /$ day for patients in the olanzapine group demonstrated statistically significantly greater improvement than in the placebo group.

Olanzapine has been included in several comparison studies for the treatment of psychosis without demonstrating any superiority over FGAs or other SGAs (risperidone, quetiapine), nor against clozapine using "high doses" (up to $30 \mathrm{mg}) .{ }^{19}$ It has been evaluated for treating disruptive behaviors (including autism) without clear benefit. ${ }^{3}$
Of all the SGAs, olanzapine remains the biggest offender overall in terms of weight gain and increases in fasting cholesterol, low-density lipoprotein, insulin, and liver transaminase levels. ${ }^{8}$ Caccia cites one study implying that dissolving tablets appear to induce less weight gain (possibly due to shorter interaction with digestive serotonin receptors, which mediate satiety). ${ }^{20}$ Adolescents have been found to gain more weight than adults on olanzapine, but have fewer adverse metabolic changes in terms of moving from normal or impaired glucose to high blood glucose and less developed borderline dyslipidemias. ${ }^{20}$

\section{Clozapine}

Clozapine is unique in its relatively low affinity for $\mathrm{D}_{2}$ receptors, making it the least risky of the SGAs in terms of extrapyramidal symptoms. Review of this medication heralds it as being the only antipsychotic with superior efficacy in comparison to both haloperidol and olanzapine in adolescents, especially in regard to early onset schizophrenic disorders and treatment-refractory psychosis. ${ }^{20,44}$ It is approved in Europe for use in youth with treatment-refractory schizophrenia.

Despite the superiority of clozapine, there are substantial incidences of adverse effects, including risk for decrease in absolute neutrophil count. ${ }^{19}$ Evidence also demonstrates significant increase in weight and BMI and elevations in cholesterol and triglycerides. ${ }^{8,20,45}$ In a recent systematic review of the efficacy and tolerability of clozapine in youth with early onset schizophrenia, the authors reported on additional adverse reactions. These included persistent sedation, seizures (average of up to 3\%), akathisia (15\%-31\%), nocturnal enuresis (up to 15\%), hypersalivation ( $80 \%-90 \%$ ), and constipation $(30 \%-50 \%)$. Of importance in regard to cardiological function, tachycardia and hypertension may persist beyond the initial months of treatment. Also, though they did not review any cases of reported myocarditis, they cautioned clinicians to remain vigilant, given other literature ${ }^{45}$ reports of rates of up to $3 \%$ in all clozapine-treated patients. In spite of the various side effects, clozapine overall was found to be tolerable, with low discontinuation rates in youth. However, because of the host of potentially fatal drug reactions, clozapine remains a third-line medication in spite of its efficacy, due to neutropenia, rare but fatal agranulocytosis, and significant weight gain and dyslipidemia. ${ }^{20}$

\section{Ziprasidone}

Ziprasidone is approved in Europe for bipolar disorder in those 10-17 years of age, but has no approval yet in the US 
for youth. Approval for the treatment of bipolar disorder in this age-group was based upon results of a 4-week, doubleblind, placebo-controlled trial $(n=149$ treatment versus $\mathrm{n}=88$ in placebo). ${ }^{46}$ Though it was approved for adults with schizophrenia, a review of recent trials showed a lack of efficacy in youth with early onset schizophrenia. ${ }^{19}$

In meta-analyses, unlike its SGA counterparts, ziprasidone has not shown any differences in weight when compared to placebo in RCTs lasting 8 weeks, and has not demonstrated adverse changes in glucose, cholesterol, or triglycerides in open-label studies up to 6 months. ${ }^{8,20}$ The same study demonstrating efficacy for bipolar disorder in youth looked at effects of a 26-week open-label extension ( $n=193$ in treatment, $n=90$ placebo) to assess tolerability and long-term effects. Ziprasidone was found to be metabolicneutral. Adverse events reported included somnolence and extrapyramidal disorders, and during open-label extension it was somnolence only. There has been controversy regarding its propensity to prolong the cardiac QT interval. Caccia reports a case study of a patient with Tourette's who died suddenly during a clinical trial of ziprasidone, but the remainder of large studies examining the risk of life-threatening arrhythmias on this medication conclude that it is likely to be clinically irrelevant..$^{20,47,48}$

\section{Paliperidone}

Paliperidone was the newest SGA approved by the FDA to treat schizophrenia in adolescents aged 12-17 years in 2011. Paliperidone is an active risperidone metabolite (9-hydroxyrisperidone), and has an osmotically controlledrelease delivery system with low peak-to-trough fluctuations. Approval for the treatment of schizophrenia came from a 6-week, double-blind, parallel-group study of participants ( $n=201)$ 12-17 years in age. ${ }^{49}$ They were randomly allocated to four groups to receive either placebo or one of three weightbased, fixed doses of paliperidone extended release $(1.5 \mathrm{mg}$, $3 \mathrm{mg}, 6 \mathrm{mg}$ or $12 \mathrm{mg}$ ). The 3-6 $\mathrm{mg}$ range resulted in significant improvement versus the other treatment groups and placebo. Weight-based dosing did not appear necessary.

Unlike its atypical counterparts, paliperidone is excreted unchanged in the urine, and is thus prescribed with caution in those with renal function impairment. ${ }^{20}$

Paliperidone was included in a meta-analysis of the newer SGAs to examine its metabolic profile. ${ }^{50}$ Data were based mostly on adults, but included the adolescent study mentioned earlier. ${ }^{49}$ Short-term and longer-term studies (up to 1 year) demonstrated statistically significant weight gain $\geq 7 \%$ when compared to placebo. In addition, there appeared to be a dose-weight response. There were no clinically relevant differences in cholesterol or triglyceride levels compared to placebo in both short- and longer-term trials, but there were relevant changes in glucose levels compared to placebo (long-term only). Likewise with weight gain, events related to EPSEs were also found to be dose-dependent, without any prolactin-related events. ${ }^{20}$ The authors of these reported findings cautioned there is still a relative paucity of long-term data needed to make decisive conclusions about its safety profile.

\section{Asenapine, lurasidone, and iloperidone}

Similar to paliperidone, the newer SGAs are limited in terms of their robust data base, especially in regard to where they land relative to older SGAs in terms of their safety profile. None of these agents appears to have published studies of use in youth with psychotic disorders.

In a meta-analysis of their metabolic profiles (paliperidone included) with short-term and longer-term trials, $\geq 7 \%$ weight gain was statistically significant for asenapine, followed by iloperidone and paliperidone, but not for lurasidone. ${ }^{50}$ However, statistically significant weight gain relative to placebo was found for all four agents. Iloperidone (short term) and asenapine (long term) were the only two agents with statistically significant differences in total cholesterol compared to placebo. Also, with the exception of iloperidone and lurasidone, there were statistical but likely irrelevant differences in high-density and low-density lipoprotein levels. In addition to paliperidone, relevant glucose changes were seen with asenapine and iloperidone. With the exception of paliperidone, none had significant changes in triglycerides.

\section{Monitoring and management of antipsychotic-induced metabolic side effects}

After an appropriate initial choice is made, the next step is to implement monitoring procedures. With the exception of paliperidone and the newer SGAs, CAMESA has made available monitoring spreadsheets to the public based on their rigorous analysis of each individual SGA safety profile (http://camesaguideline.org/information-for-doctors). ${ }^{8}$

Once significant weight gain has occurred, there are three possible interventions for management: 1) behavioral interventions, 2) switching to a more weight-neutral antipsychotic, and 3) adding a pharmacologic weight-loss agent. Though research has been done in adults examining these strategies, it is nearly absent in youth. To date, the only systematic study examining these interventions head to head in 
youth is under way as an open-label community study called Improving Metabolic Parameters of Antipsychotic Child Treatment (IMPACT). ${ }^{7}$ Because weight gain is a prominent and burdensome side effect and weight reduction has the potential also to ameliorate dyslipidemias, specific discussion of these three implementation strategies will be the primary focus.

\section{Behavioral interventions}

There has been considerable efficacy of behavioral interventions for SGA-related weight gain conducted in adults. A 2012 meta-analysis and systematic review of nonpharmacological interventions was completed in adults specifically with psychotic disorders. ${ }^{51}$ Thirteen studies were included and the interventions lasted between 2 and 12 months, with three studies including 2- to 3-month follow-up periods. Results demonstrated an effect toward the experimental group. At the end of the intervention phase, there was a $0.98 \mathrm{~kg} / \mathrm{m}^{2}$ reduction in the mean BMI. This corresponded to a $3.12 \%$ loss of initial weight. The authors noted that even though a weight loss of $5 \%-10 \%$ is the criterion for response proposed by the World Health Organization guidelines, they cited research suggesting it may be sufficient to improve weight-related complications. There were no statistically significant differences between therapeutic approaches, either individual compared with group interventions, or cognitive behavioral therapy compared with nutritional counseling.

In efforts to assess the long-term impact of behavioral interventions as a preventative strategy, a recent study investigated 2-year effects of an early behavioral intervention (EBI) designed to prevent antipsychotic-induced weight gain in mostly drug-naïve patients (96.8\% of total subjects) with FEP. ${ }^{52}$ In the control group, patients were informed about potential weight gain and advised to increase their exercise and limit food intake. Sixty-one FEP patients were randomized to receive either EBI or treatment as usual. EBI was manualized and comprised 10-14 individual sessions in which several intervention modules (assessment, psychoeducation, dietary counseling, exercise program, and behavior therapy) were implemented up to 3 months. Those in the EBI group gained significantly less weight than those allocated to routine care at intervention, with treatment effects maintained over 3 months. Differences between groups were no longer significant by 12 months, leading the authors to conclude that weight-management interventions may need to be offered for longer periods to maintain preventative effects.

In a more recent study attempting to address this issue, 291 adults from a community psychiatric rehabilitation program underwent longer-term intervention. ${ }^{53}$ Psychiatric illnesses consisted mostly of schizophrenia (58.1\%) and bipolar disorder (22.0\%). Intervention consisted of three contact types: 1) group weight-management sessions, 2) individual weight-management sessions, and 3) groupexercise sessions. Intervention was incentivized with points they could earn for small rewards and lasted for 18 months. Those in the control group received standard nutrition and physical activity information. Those in the intervention group at 18 months had a mean difference in body weight of $-3.2 \mathrm{~kg}$, with $37.8 \%$ of those in the intervention group losing $\geq 5 \%$ of initial body weight.

To date, it appears there have been no studies of utilizing behavioral interventions to prevent or reduce weight gain in pediatric populations treated with SGAs. The current intervention being utilized in the IMPACT study is using various pediatric weight-loss guidelines from the American Medical Association. ${ }^{54}$

The following conclusions and strategies may be helpful:

1. Initiating early and fairly aggressive intervention at the initiation of antipsychotics appears to prevent weight gain in the short term, but likely needs to continue for at least the first year of treatment.

2. Implementing these strategies in group settings appears to be just as effective as an individual approach, which could ease some financial burden.

3. Involving a family-based strategy is likely to ensure the best chance of success and promote healthy rolemodeling.

4. Consideration of small incentives or reward system for families may lead to greater and sustained gains to reinforce healthy, adaptive patterns.

\section{Switching agents}

Unless antipsychotics can be tapered and discontinued in the child or adolescent, it is logical to switch to a less orexigenic agent. There are complications when switching, as it may worsen outcomes and cause relapse in symptoms. Maayan and Correll summarized these findings nicely in their review of management of antipsychotic-related weight gain. ${ }^{16}$ They cited the large multicenter treatment Clinical Antipsychotic Trials of Intervention Effectiveness (CATIE), which demonstrated that patients were more likely to discontinue treatment prematurely after switching than when blindly rerandomized back to the prebaseline antipsychotic. In spite of this, studies have demonstrated significant decrease in weight and other cardiometabolic risk factors when switching to an agent with a better metabolic side-effect profile. For example, 
adult patients switched off risperidone and olanzapine lost an average of $6.9 \mathrm{~kg}$ and $9.8 \mathrm{~kg}$, respectively. The authors also point to a 2010 Cochrane review of four RCTs looking at this issue, which deemed switching to a lower-risk agent to be an effective strategy.

The IMPACT study chose as part of its switch protocol to include aripiprazole and perphenazine based on the CATIE study with adults. ${ }^{7}$ The researchers did not choose ziprasidone based on the burden of electrocardiogram monitoring. Because the problem of EPSEs remained with perphenazine, IMPACT clinicians are providing prophylactic treatment with benztropine for youth prescribed greater than $8 \mathrm{mg}$.

There is no one dosing/switching strategy recommended, and it depends on the receptor profiles of the pre- and postswitch medications and illness severity. There is concern for relapse in symptoms and other side effects associated with tapering and titrating, such as EPSEs and withdrawal, and to date these strategies have come from adult populations only.

Several tapering options suggested in clinical practice were outlined by Buckley and Correll: 1) stop the first antipsychotic abruptly and immediately start the second, 2) gradually taper the first and immediately start the second, 3) gradually taper the first while gradually starting the new, and 4) plateau cross-titrate by continuing the first antipsychotic at full dose, gradually bring the second agent to full dose, and then taper the first medication. ${ }^{55}$ Most appear to favor cross-titration strategy. The authors noted that "plateau cross-titration may be particularly useful in aripiprazole because it has a longer half-life and takes more time to build up than other agents." They warn that patients switching from a highly upregulated receptor from antagonism to one that does not block that receptor may experience acute worsening of psychosis, agitation, parkinsonism/akathisia, insomnia, and anxiety. These symptoms may be related to the new antipsychotic or may be rebound symptoms related to withdrawal from the previous drug.

There may be the temptation to assume that the switch drug is ineffective when in reality the patient could be experiencing an avoidable or treatable rebound or withdrawal phenomenon. Utilizing rescue medications to help mediate these side effects can involve adding benztropine, a benzodiazepine, or an antihistamine. The authors also suggest the use of valproic acid as coverage option for aggressive patients, though this may carry the risk of additional weight gain. Extending the crossover period is encouraged in some cases.

Above all, the authors recommended key psychoeducation points for the patient and family: 1) the new drug may or may not be as efficacious as the prior, 2) side effects may occur, and the patient should be encouraged to endure them early on as they may be transient, 3 ) withdrawal symptoms may be experienced, particularly between days 7 and 10,4) drugs should not be stopped too early, 5) heavy family support should be provided, and 6) the clinician should be readily available to discuss concerns or problems.

\section{Pharmacologic interventions}

Among all the various pharmacologic interventions for SGArelated weight, the only one to stand the test of time due to a well-established safety profile in both adults and youth is metformin. ${ }^{56,57}$ Metformin decreases hepatic gluconeogenesis and improves insulin sensitivity in the liver and muscle. It is FDA-approved for treatment of type 2 diabetes in youth 10 years of age and above.

In a 2012 systematic review, metformin was studied in randomized clinical trials $12-16$ weeks in duration (adults only), with doses ranging from 750 to $2,250 \mathrm{mg} /$ day. ${ }^{57}$ Overall, it yielded a weight loss of $2.93 \mathrm{~kg}$ relative to placebo. The authors cited two studies in youth (not included in the meta-analysis) with less consistent results. Over 16 weeks, children and adolescents aged 10-17 years who had gained more than $10 \%$ of their body weight during 12 months of treatment with an SGA (risperidone, olanzapine, or quetiapine) lost on average $4.1 \mathrm{~kg}$ relative to placebo. However, another study with a younger sample of children on risperidone had significant differences at 4 weeks, yet failed to maintain significance at 12 weeks.

A recent editorial reviewing metformin concluded that though it is statistically significant in reducing body weight, this may translate clinically into modest results. ${ }^{57}$ In addition, its effectiveness in preventing weight gain is inconclusive. There is also a curious discrepancy in psychiatric populations versus nonpsychiatric populations. In an RCT evaluating metformin versus healthy lifestyle versus combination, results favored the combination group as more effective than either intervention alone. However, metformin versus healthy lifestyle intervention in a diabetes-prevention program conducted in the general population found healthy lifestyle intervention was superior to metformin. Therefore, the authors concluded that "... metformin should likely only be added after a clinical trial of psychosocial intervention for weight loss has proven ineffective." 57

Additional findings commented upon in these studies note metformin substantially improves glycemia, triglycerides, and low-density lipid cholesterol as an additional benefit 
separate from weight loss. ${ }^{56}$ Its effects on body weight appear to act through reduction in appetite rather than increase in caloric expenditure. ${ }^{56}$ Hypoglycemia is rare, because it does not stimulate insulin production, and there have been no published pediatric cases of lactic acidosis or increase in serum lactic acid. ${ }^{57}$ Neuropathy from vitamin malabsorption can be avoided with supplementation. Frequent side effects include nausea, vomiting, and diarrhea. ${ }^{57}$

The same meta-analysis that analyzed metformin as an intervention strategy also examined other pharmacological interventions in adults. ${ }^{56}$ These included $\mathrm{H}_{2}$-receptor antagonists, topiramate, norepinephrine reuptake inhibitors (NRIs), and amantadine. Of these, there was little evidence to support the use of any of the aforementioned drugs, with the exception of NRIs (atomoxetine and reboxetine) and topiramate. NRIs are thought to act upon the hypothalamus to increase energy expenditure, in addition to sympathomimetic effects via elevating heart rate and blood pressure. However, the evidence base was small and only showed marginal benefit. Topiramate had more promising benefits in adults, demonstrating a dose-response effect. Participants of one study with new-onset psychosis took $100 \mathrm{mg}$ /day in addition to olanzapine and averted weight gain. They lost $1.3 \mathrm{~kg}$ versus a $6.0 \mathrm{~kg}$ weight gain with placebo. There are suggestions of additional benefits of further reduction in psychotic symptoms in those with schizophrenia. The mechanism of weight reduction associated with topiramate is unknown, but it causes a decreased appetite without altering energy expenditures. There are significant side effects of topiramate that can make it an undesirable choice, including paresthesia, psychomotor retardation, drooling, dizziness, headache, and mild cognitive impairment.

\section{Clinical scenario revisited}

Jordan and his parents agree that tapering and discontinuation of an antipsychotic is not recommended due to the severity of Jordan's initial presentation. They attempt behavioral modifications to address the weight gain, including monitoring his caloric intake and exercise at the gym. This does little to decrease Jordan's weight, and his constant hunger adds additional anxiety and stress. The family then opts to switch to an alternative SGA with a better metabolic side-effect profile. The cross-taper strategy is completed within 2 weeks. Midway through the switch, Jordan experiences nausea, vomiting, and stomachaches, but his psychotic symptoms do not relapse. By week 4, he and family agree he is stable, and Jordan reports less anxiety because he no longer suffers from chronic hunger. Six weeks after the initial switch, he has lost $10 \mathrm{lb}(4.5 \mathrm{~kg})$.

\section{Summary}

The present review article has focused mainly on monitoring and intervention of metabolic side effects of SGAs, given these appear to be the most common and problematic. The mechanism of metabolic side effects, particularly in youth, appears to be multifactorial, and is an area of ongoing research. In addition to metabolic side effects, individual SGAs have unique and/or greater propensity than others for additional side effects of which clinicians should be aware, and require knowledge of separate monitoring and intervention guidelines. With the exception of clozapine, the most commonly prescribed SGAs appear to have similar efficacy in treating psychosis. These medications are frequently prescribed in nonpsychotic individuals as well for behavioral management. The first priority of a clinician ought to be a rational choice of when and what to prescribe. Of second importance is deploying concurrent lifestyle interventions with SGAs to prevent weight gain. If clinicians are diligent about utilizing monitoring strategies for metabolic disturbances, such as the tables recommended and provided by CAMESA, it improves quality of patient care and further informs the direction of which intervention strategy to implement. Though the evidence base is lacking in youth, efforts are being made to systematically study three interventions in particular (behavioral, agent switching, and pharmacologic) to combat metabolic side effects. Perhaps the most important question providers can revisit before implementing one of these three strategies is whether or not an antipsychotic is necessary. This may be especially true for a nonpsychotic patient who has had a sustained period of stability and made significant gains from psychosocial interventions. A strong alliance between the provider, patient, and family is what guides the decision-making process at all stages of treatment. The common goal is ultimately to improve the quality and longevity of a young person's life.

\section{Disclosure}

The authors report no conflicts of interest in this work.

\section{References}

1. Olfson M, Blanco C, Liu SM, Wang S, Correll C. National trends in the office-based treatment of children, adolescents, and adults with antipsychotics. Arch Gen Psychiatry. 2012;69(12):1247-1256.

2. Birnbaum ML, Saito E, Gerhard T, et al. Pharmacoepidemiology of antipsychotic use in youth with ADHD: trends and clinical implications. Curr Psychiatry Rep. 2013;15(382):1-13.

3. Seida J, Schouten JR, Boylan K, et al. Antipsychotics for children and young adults: a comparative effectiveness review. Pediatrics. 2012;129(3):771-784. 
4. Correll CU. Antipsychotic use in children and adolescents: minimizing adverse effects to maximize outcomes. J Am Acad Child Adolesc Psychiatry. 2008;47(1):9-20.

5. Maayan L, Correll CU. Weight gain and metabolic risks associated with antipsychotic medications in children and adolescents. JChild Adolesc Psychopharmacol. 2011;21(6):517-535.

6. Morrato EH, Druss B, Hartung DM, et al. Metabolic testing rates in 3 state Medicaid programs after FDA warnings and ADA/APA recommendations for second-generation antipsychotic drugs. Arch Gen Psychiatry. 2010;67(1):17-24.

7. Reeves G, Keeton C, Correll CU, et al. Improving metabolic parameters of antipsychotic child treatment (IMPACT) study: rationale, design, and methods. Child Adolesc Psychiatry Ment Health. 2013;7(1):31.

8. Pringsheim T, Panagiotopoulos C, Davidson J, Ho J. Evidence-based recommendations for monitoring safety of second-generation antipsychotics in children and youth. Paediatr Child Health. 2011;16(9): 581-589.

9. Correll CU, Kane JM. One year incidence rates of tardive dyskinesia in children and adolescents treated with second-generation antipsychotics: a systematic review. J Adolesc Psychopharmacol. 2007;(17(5): 647-656.

10. Psychopharmacology Institute. Neurological side effects of antipsychotics: akathisia, dystonia and parkinsonism. 2013. Available from: http://psychopharmacologyinstitute.com/antipsychotics-videos/ neurological-side-effects-akathisia-dystonia-and-parkinsonism. Accessed May 12, 2014

11. Correll CU, Gerhard T, Olfson M. Prescribing of psychotropic medications to children and adolescents: quo vadis? World Psychiatry. 2013;12(2):127-128.

12. Correll CU, Manu P, Olshanskiy V, Napolitano B, Kane JM, Malhotra AK. Cardiometabolic risk of second-generation antipsychotics during first-time use in children and adolescents. JAMA. 2009;302(16): 1765-1773.

13. McVoy M, Findling R. Clinical Manual of Child and Adolescent Psychopharmacology. 2nd ed. Arlington (VA): American Psychiatric Publishing; 2013.

14. De Hert M, Dobbelaere M, Sheridan EM, Cohen D, Correll CU. Metabolic and endocrine adverse effects of second-generation antipsychotics in children and adolescents: a systematic review of randomized, placebo controlled trials and guidelines for clinical practice. Eur Psychiatry. 2011;26(3):144-158.

15. Centers for Disease Control and Prevention. Childhood obesity facts 2014. Available from: http://www.cdc.gov/obesity/data/childhood.html. Accessed May 12, 2014.

16. Maayan L, Correll CU. Management of antipsychotic-related weight gain. Expert Rev Neurother. 2010;10(7):1175-1200.

17. Sikich L, Frazier JA, McClellan J, et al. Double-blind comparison of first- and second-generation antipsychotics in early-onset schizophrenia and schizoaffective disorder: findings from the treatment of early-onset schizophrenia spectrum disorders (TEOSS) study. Am J Psychiatry. 2008;165(11):1420-1431.

18. Sarkar S, Grover S. Antipsychotics in children and adolescents with schizophrenia: a systematic review and meta-analysis. Indian $J$ Pharmacol. 2013;45(5):439-446.

19. Correll C, Kratochvil C, March JS. Developments in pediatric psychopharmacology: focus on stimulants, antidepressants, and antipsychotics. J Clin Psychiatry. 2011;72(5):655-670.

20. Caccia S. Safety and pharmacokinetics of atypical antipsychotics in children and adolescents. Pediatr Drugs. 2013;(15):217-233.

21. Correll CU, Sheridan EM, DelBellow MP. Antipsychotic and mood stabilizer efficacy and tolerability in pediatric and adult patients with bipolar I mania: a comparative analysis of acute, randomized, placebocontrolled trials. Bipolar Disord. 2010;12(2):116-141.

22. Castro-Fornieles J, Parellada M, Gonzalez-Pinto A, et al. The child and adolescent first-episode psychosis study (CAFEPS): design and baseline results. Schizophr Res. 2007;91(1-2):226-237.

23. Haas M, Unis AS, Armenteros J, Copenhaver MD, Quiroz JA, Kushner SF. A 6-week, randomized, double-blind, placebo-controlled study of the efficacy and safety of risperidone in adolescents with schizophrenia. J Child Adolesc Psychopharmacol. 2009;19(6):611-621.
24. Haas M, Eerdekens M, Kushner S, et al. Efficacy, safety and tolerability of two dosing regimens in adolescent schizophrenia: double-blind study. Br J Psychiatry. 2009;194(2):158-64.

25. Haas M, DelBello MP, Pandina G, et al. Risperidone for the treatment of acute mania in children and adolescents with bipolar disorder: a randomized, double-blind, placebo-controlled study. Bipolar Disord. 2009;11(7):687-700.

26. Palvuluri MN, Henry DB, Findling RL, et al. Double-blind randomized trial of risperidone versus divalproex in pediatric bipolar disorder. Bipolar Disord. 2010;12(6):593-605.

27. Shea S, Turgay A, Carroll A, et al. Risperidone in the treatment of disruptive behavioral symptoms in children with autistic and other pervasive developmental disorders. Pediatrics. 2004;114(5): 634-641.

28. Pandina GJ, Bossie CA, Youssef E, Zhu Y, Dunbar F. Risperidone improves behavioral symptoms in children with autism in a randomized, double-blind, placebo-controlled trial. JAutism Dev Disord. 2007;37(2): 367-373.

29. Ghanizadeh A, Sahraeizadeh A, Berk MA. Head-to-head comparison of aripiprazole and risperidone for safety and treating autistic disorders, a randomized double blind clinical trial. Child Psychiatry Hum Dev. 2014;45(2):185-192.

30. Margari L, Matera E, Craig F, et al. Tolerability and safety profile of risperidone in a sample of children and adolescents. Int Clin Psychopharmacol. 2013;28(4):177-183.

31. Findling RL, Robb A, Nyilas M, et al. A multiple-center, randomized, double-blind, placebo-controlled study of oral aripiprazole for treatment of adolescents with schizophrenia. Am J Psychiatry. 2008;165(11): 1432-1441.

32. Findling RL, Nyilas M, Forbes RA, et al. Acute treatment of pediatric bipolar I disorder, manic or mixed episode, with aripiprazole: a randomized, double-blind, placebo-controlled study. J Clin Psychiatry. 2009;70(10):1441-1451.

33. Marcus RN, Owen R, Kamen L, et al. A placebo-controlled, fixeddose study of aripiprazole in children and adolescents with irritability associated with autistic disorder. J Am Acad Child Adolesc Psychiatry. 2009;48(11):1110-1119.

34. Owen R, Sikich L, Marcus RN, et al. Aripiprazole in the treatment of irritability in children and adolescents with autistic disorder. Pediatrics. 2009;124(6):1533-1540.

35. Findling RL, Mankoski R, Timko K. Randomized controlled trial investigating the safety and efficacy of aripiprazole in the long-term maintenance treatment of pediatric patients with irritability associated with autistic disorder. J Clin Psychiatry. 2014;75(1):22-30.

36. Findling RL, McKenna K, Earley WR, Stankowski J, Pathak S. Efficacy and safety of quetiapine in adolescents with schizophrenia investigated in a 6-week, double-blind, placebo-controlled trial. J Child Adolesc Psychopharmacol. 2012;22(5):327-342.

37. Arango C, Robles O, Parellada M, et al. Olanzapine compared to quetiapine in adolescents with a first psychotic episode. Eur Child Adolesc Psychiatry. 2009;18(7):418-428.

38. Jensen J, Kumra S, Leitten W, et al. A comparative pilot study of second-generation antipsychotics in children and adolescents with schizophrenia-spectrum disorders. J Child Adolesc Psychopharmacol. 2008;18(4):317-326.

39. Pathak S, Findling RL, Earley WR, Acevedo LD, Stankowski J, Delbello MP. Efficacy and safety of quetiapine in children and adolescents with mania associated with bipolar I disorder: a 3-week, double-blind, placebo-controlled trial. J Clin Psychiatry. 2013;74(1): e100-e109.

40. DelBello MP, Kowatch RA, Adler CM, et al. A double-blind randomized pilot study comparing quetiapine and divalproex for adolescent mania. J Am Acad Child Adolesc Psychiatry. 2006;45(3): 305-313.

41. Connor DF, McLaughlin TJ, Jeffers-Terry M. Randomized controlled pilot study of quetiapine in the treatment of adolescent conduct disorder. J Child Adolesc Psychopharmacol. 2008;18(2):140-156. 
42. Kryzhanovskaya L, Schulz SC, McDougle C, et al. Olanzapine versus placebo in adolescents with schizophrenia: a 6-week, randomized, double-blind, placebo-controlled trial. J Am Acad Child Adolesc Psychiatry. 2009;48(1):60-70.

43. Tohen M, Kryzhanovskaya L, Carson G, et al. Olanzapine versus placebo in the treatment of adolescents with bipolar mania. Am J Psychiatry. 2007;164(10):1547-1556.

44. Agid O, Remington G, Kapur S, Arenovich T, Zipursky RB. Early use of clozapine for poorly responding first-episode psychosis. J Clin Psychopharmacol. 2007;27(4):369-373.

45. Schneider C, Corrigall R, Hayes D, Kyriakopoulos M, Frangou S. Systematic review of the efficacy and tolerability of clozapine in the treatment of youth with early onset schizophrenia. Eur Psychiatry. 2014;29(1):1-10.

46. Findling RL, Cavuş I, Pappadopulos E, et al. Efficacy, long-term safety, and tolerability of ziprasidone in children and adolescents with bipolar disorder. J Child Adolesc Psychopharmacol. 2013;23(8):545-557.

47. Correll CU, Lops JD, Figen V, Malhotra AK, Kane JM, Manu P. QT interval duration and dispersion in children and adolescents treated with ziprasidone. J Clin Psychiatry. 2011;72(6):854-860.

48. Strom BL, Eng SM, Faich G, et al. Comparative mortality associated with ziprasidone and olanzapine in real-world use among 18,154 patients with schizophrenia: the Ziprasidone Observational Study of Cardiac Outcomes (ZODIAC). Am J Psychiatry. 2011;168(2):193-201.

49. Singh J, Robb A, Vijapurkar U, Nuamah I, Hough D. A randomized, double-blind study of paliperidone extended-release in treatment of acute schizophrenia in adolescents. Biol Psychiatry. 2011;70(12):1179-1187.

50. DeHert M, Weiping Y, Detraux J, Sweers K, van Winkel R, Correll C. Body weight and metabolic adverse effects of asenapine, iloperidone, lurasidone and paliperidone in the treatment of schizophrenia and bipolar disorder: a systematic review and exploratory meta-analysis. CNS Drugs. 2012;26(9):733-759.
51. Bonfioli E, Berti L, Goss C, Muraro F, Burti L. Health promotion lifestyle interventions for weight management in psychosis: a systematic review and meta-analysis of randomized controlled trials. $B M C$ Psychiatry. 2012;12:78.

52. Alvarez-Jiménez M, Martínez-García O, Pérez-Iglesias R, Ramírez ML, Vázquez-Barquero JL, Crespo-Facorro B. Prevention of antipsychoticinduced weight gain with early behavioural intervention in first-episode psychosis: 2-year results of a randomized controlled trial. Schizophr Res. 2010;116(1):16-19.

53. Daumit G, Dalcin AT, Jerome GJ, et al. A behavioral weight-loss intervention in persons with serious mental illness. $N$ Engl J Med. 2013;388(17):1594-1602

54. American Medical Association. Recommendations on the assessment, prevention, and treatment of childhood obesity. 2007. Available from: http://www.ama-assn.org/ama/pub/physician-resources/public-health/ promoting-healthy-lifestyles/obesity/childhood-obesity/assessmentprevention-treatment.page?. Accessed May 12, 2014.

55. Buckley PF, Correll CU. Strategies for dosing and switching antipsychotics for optimal clinical management. J Clin Psychiatry. 2008; 69 Suppl 1:4-17.

56. Fiedorowicz JG, Miller DD, Bishop JR, Calarge CA, Ellingrod VL, Haynes WG. Systematic review and meta-analysis of pharmacological interventions for weight gain from antipsychotics and mood stabilizers. Curr Psychiatry Rev. 2012;8(1):25-36.

57. Correll C, Sikich L, Reeves G, Riddle M. Metformin for antipsychoticrelated weight gain and metabolic abnormalities: when, for whom, and for how long? Am J Psychiatry. 2013;120(9):947-952.
Adolescent Health, Medicine and Therapeutics

\section{Publish your work in this journal}

Adolescent Health, Medicine and Therapeutics is an international, peer-reviewed, open access journal focusing on health, pathology, and treatment issues specific to the adolescent age group. All aspects of health maintenance, preventative measures and disease treatment interventions are addressed within the journal and practitioners from

\section{Dovepress}

all disciplines are invited to submit their work as well as healthcare researchers and patient support groups.. The manuscript management system is completely online and includes a very quick and fair peerreview system. Visit http://www.dovepress.com/testimonials.php to read real quotes from published authors. 